\title{
Syringohydromyelia in Patients with Chiari I Malformation: A Retrospective Analysis
}

\author{
(D).A. Gad and DD.M. Yousem
}

\begin{abstract}
BACKGROUND AND PURPOSE: The association of syringohydromyelia with Chiari I malformation has a wide range, between $23 \%$ and $80 \%$ of cases in the current literature. In our experience, this range might be overestimated compared with our observations in clinical practice. Because there is an impact of Chiari I malformation-associated syringohydromyelia on morbidity and surgical intervention, its diagnosis is critical in this patient population. Identifying related variables on the basis of imaging would also help identify those patients at risk of syrinx formation during their course of disease.
\end{abstract}

MATERIALS AND METHODS: We performed a retrospective analysis of the MR imaging studies of 108 consecutive cases of Chiari I malformation. A multitude of factors associated with syrinx formation were investigated, including demographic, morphometric, osseous, and dynamic CSF flow evaluation.

RESULTS: Thirty-nine of 108 (36.1\%) patients with Chiari I malformation had syringohydromyelia. On the basis of receiver operating characteristic curve analysis, a skull base angle (nasion-sella-basion) of $135^{\circ}$ was found to be a statistically significant classifier of patients with Chiari I malformation with or without syringohydromyelia. Craniocervical junction osseous anomalies $(O R=4.3, P=$ $.001)$ and a skull base angle of $>135^{\circ}(\mathrm{OR}=4.8, P=.0006)$ were most predictive of syrinx formation. Pediatric patients (younger than 18 years of age) who developed syringohydromyelia were more likely to have associated skull base osseous anomalies than older individuals $(P=.01)$.

CONCLUSIONS: Our findings support evidence of the role of foramen magnum blockage from osseous factors in the development of syringohydromyelia in patients with Chiari I malformation.

ABBREVIATION: $\mathrm{CMI}=$ Chiari I malformation

T he association of syringohydromyelia with Chiari I malformation $(\mathrm{CMI})$ is estimated in the literature, with a wide range between $23 \%$ and $80 \%$ of cases. ${ }^{1-5}$ The heterogeneity of values may imply either inconsistent methodologic criteria for defining CMI with discrepancy of measurements or a possible overlap among cases with true syrinx and central canal dilation, and those with cord edema and a clinical presyrinx state. Moreover, the true prevalence of syrinx in this population remains poorly defined due to factors related to characteristics of the studied populations. Selecting only patients who have myelopathic symptoms may

Received July 14, 2016; accepted after revision May 6, 2017.

From the Division of Neuroradiology (K.A.G., D.M.Y.), The Russell H. Morgan Department of Radiology and Radiological Science, The Johns Hopkins Medical Institutions, Baltimore, Maryland; and Radiology Department (K.A.G.), Faculty of Medicine, Suez Canal University, Ismailia, Egypt.

Please address correspondence to David M. Yousem, MD, MBA, Johns Hopkins Medical Institutions, 600 N. Wolfe St, Phipps B100F, Baltimore, MD 21287; e-mail: dyousem1@jhu.edu

http://dx.doi.org/10.3174/ajnr.A5290 falsely increase the prevalence. Similar selection bias can also occur when including only patients who are referred for neurosurgical consultation because of spinal pathology.

A large-scale survey for the Pediatric Section of the American Association of Neurologic Surgeons concluded that syringohydromyelia is considered an indication for surgery in CMI. ${ }^{6}$ Recent DTI studies have shown significantly decreased fractional anisotropy values at the level of a syrinx, which appeared more evident in symptomatic patients. ${ }^{7}$ The critical association of syringohydromyelia secondary to CMI with regard to surgical intervention makes early diagnosis crucial in choosing the management plan for these patients.

In the current study, we sought to determine the prevalence of syringohydromyelia associated with symptomatic CMI by a retrospective analysis of $108 \mathrm{MR}$ imaging cases diagnosed at our institution with the most widely accepted diagnostic criteria. A multitude of demographic, morphometric, osseous, and functional neuropathologic factors associated with the condition were 


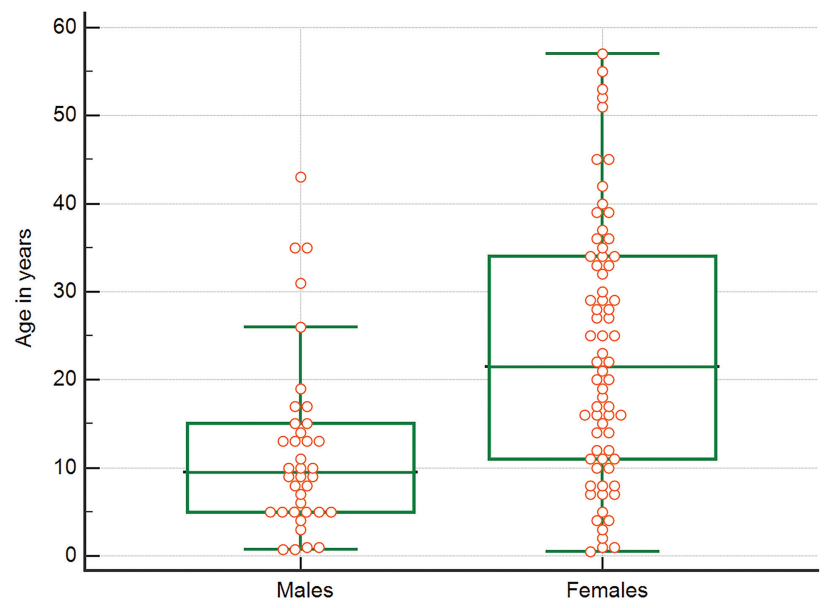

FIG 1. A graph plotting age and sex demographics of all study subjects.

investigated to better understand the pathophysiology of CMIassociated syringohydromyelia. We hypothesized that skull base anomalies may be related to syrinx formation in symptomatic patients with CMI.

\section{MATERIALS AND METHODS}

Following institutional review board approval and with Health Insurance Portability and Accountability Act compliance, 108 consecutive patients with the MR imaging diagnosis of CMI were retrospectively identified in radiology records at the Johns Hopkins Medical institution. A request form was submitted to search for patients with CMI in radiology reports with the following keywords: Chiari 1, Chiari I, CM1, and CMI.

Inclusion criteria were herniation of at least 1 cerebellar tonsil $\geq 5 \mathrm{~mm}$ below the McRae line (basion-to-opisthion) on sagittal T1-weighted images, and supporting clinical findings including the following: 1) posterior headache worse with the Valsalva maneuver, coughing, sneezing, or exercise; 2) neck pain, tingling, numbness, or burning; 3) generalized weakness or unrelenting fatigue; 4) dizziness worse with lying down; 5) peripheral motor/ sensory deficit; 6) sleep apnea; or 7) lower cranial neuropathy. Thirty-seven males and 70 females with a median age of 15.5 years (95\% CI, 12.3-19.0 years) were included from January 2014 to May 2016 (Fig 1). Any tonsillar herniation secondary to a spaceoccupying lesion was excluded. Exclusion criteria also included previous decompressive, cranial, or spinal surgeries. MR images were evaluated, including sagittal T1, axial T1, and axial T2 of the brain and T1 (sagittal and axial) and T2 (sagittal and axial) of the spine. Dynamic phase-contrast CSF flow studies were also evaluated when available.

After we excluded 7 studies due to poor technical quality, only 69 patients with technically accepted phase-contrast images at a velocity-encoding threshold of $5 \mathrm{~cm} / \mathrm{s}$ were analyzed. CSF flow was evaluated qualitatively on the basis of phase-contrast cine images. A normal flow pattern must be a clear bidirectional flow signal: caudal and cranial (black and white with the cardiac cycle) at the ventral and dorsal columns within the foramen magnum. Any deviation from this strict criterion was considered abnormal CSF flow.

Two blinded subspecialty-certified neuroradiologists (with

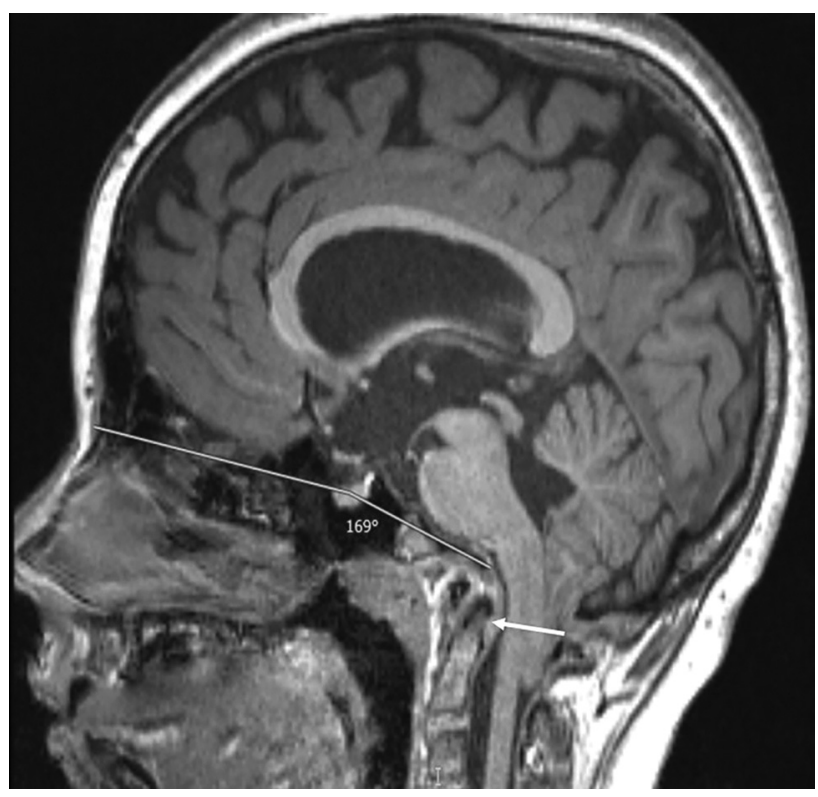

FIG 2. A 12-year-old male patient with CMI. The skull base angle from the nasion to the center of the pituitary fossa to the clivus (anterior wall of the foramen magnum) measures $169^{\circ}$. The odontoid tip is retroverted; this feature results in compression on the cervicomedullary junction (arrow).

15 and 11 years of neuroradiology practice) read the cases followed by a third reading by an expert subspecialty-certified neuroradiologist (with 27 years of neuroradiology practice) if there was any interrater discordance. The main dependent variable was the presence of syringohydromyelia. Any measurable intramedullary sharply demarcated fluid cavity of $>2-\mathrm{mm}$ width in the axial plane that followed CSF signal in both $\mathrm{T} 1$ and T2 imaging was considered a syrinx. We identified the following independent variables: degree (in millimeters) of tonsillar herniation, the presence of hydrocephalus, the location and size of the syrinx, and associated craniocervical osseous anomalies including the following: 1) platybasia defined as abnormal flattening of the skull base with a basal angle measuring $>143^{\circ}$ between intersecting lines from the nasion to the sella (in the center of the pituitary gland and from the sella to the anterior margin of the foramen magnum) (Fig 2) ${ }^{8}$; 2) a retroverted odontoid defined as a posterior inclination of the odontoid relative to the body of $\mathrm{C} 2$ with a retroversion angle measuring $<70^{\circ}$ (Fig 3$)^{9}$; 3) a short hypoplastic clivus defined as $<1.5-\mathrm{cm}$ clival length measured from the sphenoclival synchondrosis to the basion ${ }^{10}$; and 4) basilar invagination defined as a protrusion of the odontoid tip of at least $5 \mathrm{~mm}$ above the Chamberlain line between the posterior end of the hard palate and the posterior lip of the foramen magnum (Fig 4). ${ }^{10}$ All measurements were made with the VitreaView platform 2010-2016 (Vital Images, Minnetonka, Minnesota), with electronic calipers and electronic measurement of angulation.

\section{Statistical Analysis}

We calculated the prevalence of syringohydromyelia in our sample. We examined the likelihood of an association of the variables defined above with the occurrence of syringohydromyelia using $\chi^{2}$ analysis. For continuous variables, receiver operating charac- 


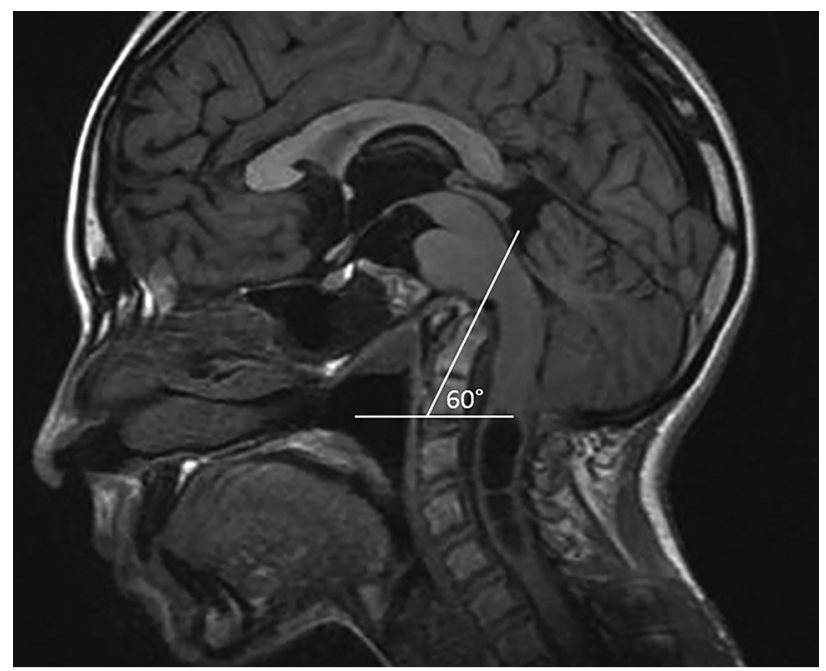

FIG 3. Sagittal TI-weighted MR image demonstrating an abnormally small odontoid retroversion angle $\left(\sim 60^{\circ}\right)$ between a horizontal line parallel to the inferior endplate of $\mathrm{C} 2$ and the long axis of the odontoid process passing through its tip in a child with CMI and associated cervical syrinx.

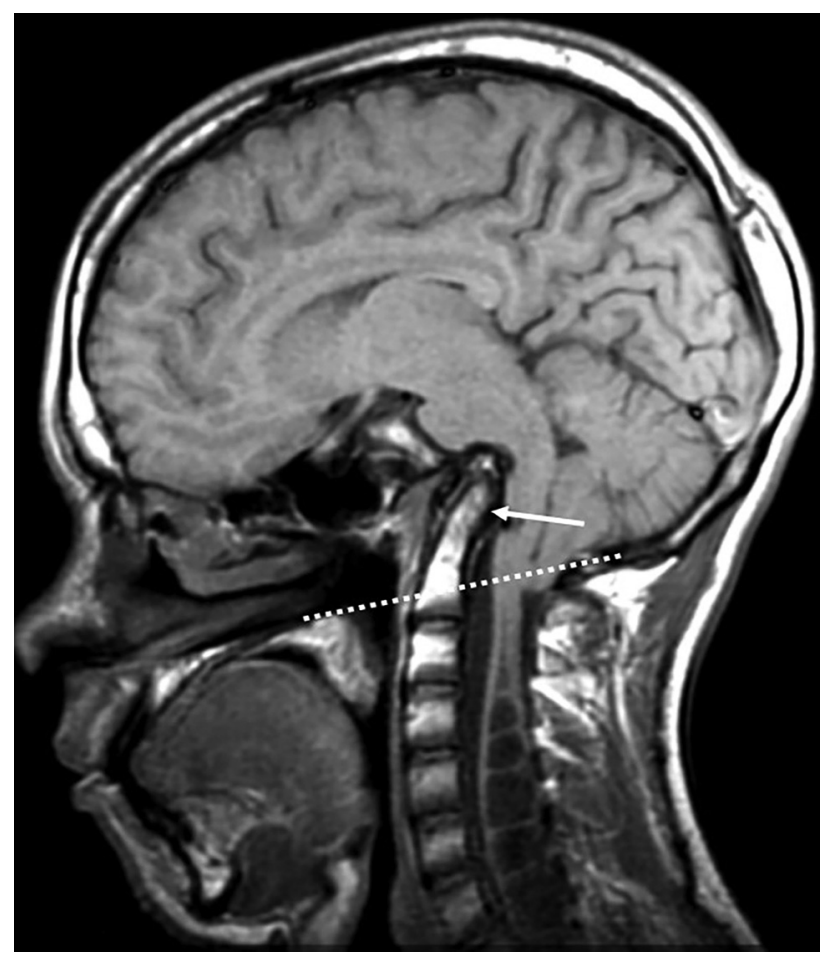

FIG 4. Sagittal $\mathrm{Tl}$ at the level of craniocervical junction in an 18-yearold male patient with CMI showing a retroverted odontoid (arrow) with its tip protruding into the foramen magnum $22 \mathrm{~mm}$ above the Chamberlain line (dotted line), resulting in basilar invagination and anterior brain stem compression. Note multilevel syrinx formation of the upper cord. The clivus (not completely shown) is short and deformed.

teristic curves were generated to identify cutoff points with the best performance of sensitivity and specificity. At these cutoff points, logistic regression analysis was performed to identify the best-fitting model that affects the occurrence of syringohydromyelia in patients with CMI. Odds ratios were calculated. All statistical tests were performed by using the MedCalc statistical soft-
Frequency of syrinx formation according to location

\begin{tabular}{lc}
\hline Syrinx Location $(\boldsymbol{n}=\mathbf{3 9})$ & No. \\
\hline Cervicothoracic & 23 \\
Cervical & 9 \\
Thoracic & 6 \\
Whole cord & 1 \\
\hline
\end{tabular}

ware package for Windows, Version 16.4.3-2016, (MedCalc Software, Mariakerke, Belgium).

\section{RESULTS}

Syringohydromyelia was present in 39 (36.1\%) of 108 symptomatic patients with CMI (Table). Hydrocephalus was present in only $7 / 108$ patients $(6.5 \%)$; 3 of 7 had no syringohydromyelia. No statistically significant association was found between the patient age or sex and syrinx formation. Among our total sample of 108 symptomatic patients with CMI, 30 had $\geq 1$ bony anomaly identified at the craniocervical junction as follows: retroverted odontoid $\left(n=23\right.$, with the retroversion angle ranging from $59^{\circ}$ to $\left.68^{\circ}\right)$; platybasia ( $n=15$, with the skull base angle ranging from 145 to $164^{\circ}$ ); short clivus ( $n=7$, with clival length ranging from 9 to 43 $\mathrm{mm})$; and basilar invagination ( $n=6$, with the protrusion distance above the Chamberlain line ranging from 5 to $22 \mathrm{~mm}$ ). The presence of any type of craniocervical bony anomaly was statistically associated with syringohydromyelia $(P=.001)$. A skull base angle at a cutoff point of $135^{\circ}$ was identified by the receiver operating characteristic curve as having the best performance for the prediction of syringohydromyelia $(P=.02)$ with a sensitivity of $50 \%$ and a specificity of $82.6 \%$ (Fig 5 ). Above this cutoff point $\left(>135^{\circ}\right)$, there was a strong $\chi^{2}$ association with syrinx formation $(P=.0002)$. With univariate logistic regression analysis, craniocervical junction osseous anomalies (odds ratio $=4.3, P=.001$ ) and a skull base angle of $>135^{\circ}$ (odds ratio $=4.8, P=.0006$ ) were the most predictive of syrinx formation. With multivariate analysis, the only predictive variable was a skull base angle of $>135^{\circ}$.

Among patients with syringohydromyelia $(n=39)$, the patient age was associated with craniocervical anomalies based on logistic regression $(P=.01)$. Receiver operating characteristic curve analysis showed a cutoff point of 18 years as patients younger than 18 years of age with syringohydromyelia were more likely to have associated craniocervical bony anomalies compared with older individuals $(P=.04)$.

There was no statistically significant association between the degree of tonsillar herniation or abnormal phase-contrast CSF flow at the foramen magnum and syrinx formation.

Of our 39 cases of syringohydromyelia, 25 had a large syrinx with the widest dimension on axial scans of $>6 \mathrm{~mm}$. The smallest syrinx size encountered in 1 case measured $3 \mathrm{~mm}$ in width. However, no statistically significant association could be found between the size of syrinx and any of the tested variables.

\section{DISCUSSION}

\section{Prevalence of Syringohydromyelia}

Identifying syrinx formation in patients with symptomatic CMI is critical in working up their management plan. In our descriptive study of 108 symptomatic patients who were clinically diagnosed with CMI, syringohydromyelia was present in 39 (36.1\%) pa- 

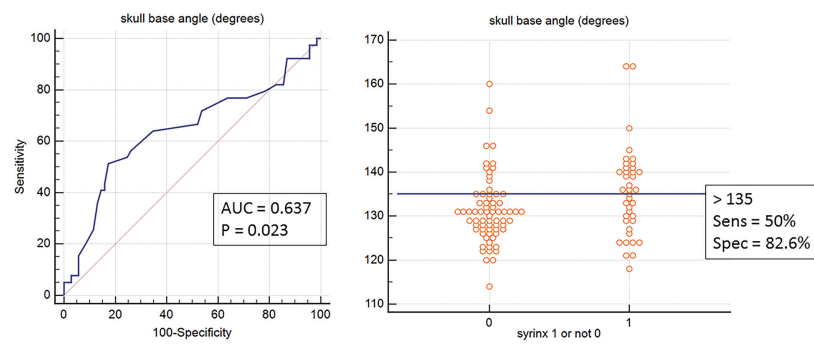

FIG 5. Receiver operating characteristic curve (left) and plot graph (right) demonstrating the skull base angle in the prediction of syrinx formation among our total sample $(n=108)$. AUC indicates area under the curve; Sens, sensitivity; Spec, specificity.

tients. We believe that our prevalence is not affected by any selection bias that may falsely overestimate or underestimate the true figures because our patients were identified by MR imaging as well as clinical criteria in a specific 2014-2016 timeframe, including all referred individuals based on MR imaging findings and clinical assessment. They were not selected from neurosurgical archives or data bases in a trial, to avoid commonly encountered overestimation of syringohydromyelia in studies in which patients were surgical candidates. Our sample also included those patients who were incidentally diagnosed while undergoing MR brain imaging for any other reason and then referred for clinical consultation and diagnosed as having CMI based on confirming clinical signs and symptoms. Patients with secondary CMI such as those with an intracranial space-occupying lesion were also excluded.

Several studies have been attempted, with different criteria, to explore the imaging prevalence of syringohydromyelia among patients with CMI. In a study by Strahle et al, ${ }^{3}$ most patients underwent MR imaging of the cervical spine and almost half of them underwent full spine imaging, resulting in $22.9 \%$ of patients with $\mathrm{CMI}$ having a syrinx detected. Aitken et $\mathrm{al}^{11}$ identified spinal syringes in $12 \%$ of children with CMI in their study; the authors relied on reviewing only radiology reports in many patients without having access to their images.

\section{Craniocervical Bony Anomalies}

In our study, the presence of craniocervical bony anomalies was statistically associated with syringohydromyelia. A retroverted odontoid with an angle of $<70^{\circ}$ was found to be the most common developmental osseous anomaly $(n=23)$ of the craniocervical junction among our series, while basilar invagination was present in only 6 cases. With logistic regression, craniocervical junction osseous anomalies and a skull base angle of $>135^{\circ}$ were most predictive of syrinx formation. Since 1896, when Arnold Chiari highlighted insufficient bone growth and insufficient enlargement of portions of the skull during development as additional mechanisms that cause increased intracranial pressure and subsequent tonsillar herniation, it has been accepted that developmental morphometric anomalies of the posterior fossa are linked to CMI.

In the classic study on 364 patients with CMI by Milhorat et $\mathrm{al},{ }^{1}$ in which syringohydromyelia was found in $65 \%$ of patients, the authors highlighted commonly associated problems, including scoliosis (42\%), a retroflexed odontoid process (26\%), basilar invagination (12\%), and varying degrees of bony dysplasia of the posterior cranial fossa based on volumetric calculations. Vega et $\mathrm{al}^{12}$ studied 42 patients with CMI compared with 46 controls and found that patients with CMI had shorter clivus lengths and a shorter Chamberlain line. The concept of complex Chiari malformation is being widely accepted in the neurosurgery literature. A complex severe craniocervical anomaly would add more burden to the disease. ${ }^{13}$ We believe that a complex Chiari malformation might represent a separate entity of the disease that requires a different surgical approach. Brockmeyer ${ }^{14}$ defined the complex Chiari malformation as "cerebellar tonsil herniation combined with one or more of the following radiographic findings: brain stem herniation through the foramen magnum (Chiari 1.5 malformation), medullary kink, retroflexed odontoid, abnormal clival-cervical angle, occipitalization of the atlas, basilar invagination, syringohydromyelia or scoliosis." The authors differentiated patients with complex Chiari malformation from those with typical CMI as being more likely to require advanced surgical interventions other than standard suboccipital decompression.

In another study by Moore and Moore, ${ }^{15}$ odontoid retroflexion, craniovertebral junction osseous anomalies, and syringohydromyelia were more often observed in patients with complex Chiari malformation than in those with typical CMI. These observations are in parallel with our findings. Our odds ratios were 4.8 and 4.3 for craniocervical anomalies and a skull base angle of $>135^{\circ}$, respectively, in the prediction of syringohydromyelia. These findings might give importance to the variant of complex Chiari malformation as a special research entity and should be a point of emphasis in the radiology report when these findings are present. The position of the obex was described in the same study by Moore and Moore ${ }^{15}$ as a characteristic imaging feature that was present in all complex cases. However, we did not evaluate the obex level in our study due to interobserver variability.

CSF flow studies indicated that the tonsils in CMI partially obstruct the free movement of CSF across the foramen magnum. Normally, the cardiac systole delivers blood to the brain; most of the new volume is absorbed by the venous system of the brain, however, during systole; and about $0.75-1.0 \mathrm{~mL}$ of CSF gets rapidly moved from the cisterna magna across the subarachnoid space and into the spinal subarachnoid space at the level of the foramen magnum. This extra amount of spinal CSF should move back to the cranial space during diastole. Blocking this backward rapid movement of CSF partially entraps the spinal intrathecal space, resulting in reduced compliance in the spinal CSF space. The tonsillar movement is against a partially entrapped CSF space with reduced compliance and increased intrathecal pressure. It is the complex interplay between clivus inclination and odontoid angulation that can create a point of mechanical stress and tension leading to anterior brain stem compression.

The presence of associated skull base anomalies makes CMI defined by neurosurgeons a "complex" Chiari malformation, which requires more advanced surgical decompressive approaches. According to Bollo et al, ${ }^{13}$ the presence of basilar invagination puts the patient at risk of craniocervical fusion. In our study, we evaluated the presence of craniocervical kyphosis by identifying platybasia and odontoid retroversion and by measuring the skull base angle (intersecting lines from the nasion to the sella and from the sella to the anterior margin of the foramen 
magnum). A normal angle lies between $125^{\circ}$ and $143^{\circ}$. Although an angle of $>143^{\circ}$ is required to quantitatively diagnose platybasia, we found that higher angles are more commonly associated with syrinx formation using logistic regression among our cases of CMI. Our findings are in full support of the suggested pathophysiology of CMI, in which an obtuse angle results in an upward tilt of the clivus with a relatively higher location of the opisthion. This aberrant osseous configuration is associated with 2 abnormal morphometric changes: 1) narrowing or ventral compromise to the foramen magnum with corresponding downstream CSF flow resulting in syrinx formation, and 2) a smaller posterior fossa volumetric capacity with corresponding cerebellar herniation and crowding of structures at the already compromised foramen magnum, leading to more blockage.

A cutoff point of $135^{\circ}$ was statistically significant, showing a relatively high specificity of $82.6 \%$ in predicting an associated syringohydromyelia. Although its sensitivity was not high (50\%), there were only $12 / 69(17.4 \%)$ patients without syrinx who showed a skull base angle above $135^{\circ}$. Sgouros et al ${ }^{16}$ noted that skull base angles were larger in 30 patients with CMI compared with controls. The authors used a modified angle with the dorsum sella as a reference midpoint for intersecting lines. Their angle was slightly higher than ours where lines intersect more inferiorly in the pituitary center; however, in their results, the presence of syringohydromyelia was also associated with a larger angle $\left(151^{\circ}\right)$ compared with CMI without syringohydromyelia $\left(145^{\circ}\right)$. We believe that measuring the skull base angle in CMI can help identify those patients at risk of developing syrinx $(\mathrm{OR}=4.8, P=.0006)$.

\section{Impaired CSF Flow by Tonsillar Herniation}

Disturbed CSF flow dynamics remain a standard phenomenon by which patients with CMI can develop syringohydromyelia. Bunck et $\mathrm{al}^{17}$ revealed pronounced alteration of CSF flow dynamics with $4 \mathrm{D}$ phase-contrast quantitative MR imaging in 12 patients with CMI with syringohydromyelia compared with 8 without it. In our study, we could not establish a statistically significant association between impaired CSF flow in cine phase-contrast MR imaging and syrinx formation. The association between the degree of mechanical blockage and the formation of syrinx has been debatable in the literature. Our finding of the lack of association may shed light on a noncompressive etiology of syringohydromyelia.

Schuster et $\mathrm{al}^{18}$ presented a systematic review of residual syringohydromyelia after decompressive surgery, in which the rate of postoperative persistent syrinx ranged between $0 \%$ and $22 \%$, raising the possibility of a noncompressive etiology in the development of syrinx. We believe that CSF flow dynamics at the foramen magnum is a complicated process that would have been more accurately evaluated if quantitative indicators were considered. Due to this technical limitation of our study besides the relatively small number of cases with available technically acceptable phase-contrast MR images (69/108), a clear interpretation of these findings was not possible.

Clarke et $\mathrm{al}^{19}$ presented a computational model in CMI, in which the presence and absence of syringohydromyelia were compared. This study introduced an advanced understanding of the pressure gradient and peak pressure timing differences between CMI with or without syringohydromyelia. The authors found that the dynamic profile of subarachnoid spinal pressure relative to the cardiac cycle (in which intraspinal arteriolar pressure varies) is more critical in regard to the chance of fluid accumulation and the development of a presyrinx or syrinx state. ${ }^{19}$

The association between the degree of mechanical blockage and the formation of syrinx has been debatable in the literature. Although the degree of tonsillar herniation in our results gives an insight into the mechanical perspective of CSF flow compromise at the foramen magnum, it is still insufficient to predict syringohydromyelia on the basis of how far cerebellar tonsils have descended, possibly because of the complex mechanical-versusnonmechanical algorithms determining the pathogenesis of the condition.

\section{Study Limitations}

Our study had a few technical limitations related to the partial inhomogeneity of the data (such as fewer CSF flow studies) as well as the cross-sectional design with a lack of longitudinal follow-up. The development of syringohydromyelia in CMI is a dynamic process. Further longitudinal studies are needed to follow up patients with CMI, trying to identify different trends in relation to syrinx formation in those patients and how these trends could be affected by the degree of mechanical blockage and the age of the patient. Quantitative assessment of CSF flow would also represent a valid parameter compared with the qualitative method we used. Assessment of the obex level by quantitative measurement of its position relative to the foramen magnum would have been an important variable that we recommend in future studies because it might be linked to the diagnosis of complex Chiari malformation besides other craniocervical anomalies.

\section{CONCLUSIONS}

Our prevalence of syringohydromyelia associated with CMI $(36.1 \%)$ lies in the low range compared with existing values in the literature. In view of the statistically significant association between syrinx formation and craniocervical osseous abnormalities, our findings support the role of developmental foramen magnum compromise in the pathophysiology of CMIrelated syringohydromyelia.

Disclosures: David M. Yousem—UNRELATED: Expert Testimony: medicolegal cases, self-employed; Payment for Lectures Including Service on Speakers Bureaus: American College of Radiology Education Center course director*; Royalties: Elsevier for 4 books; Payment for Development of Educational Presentations: CMEInfo.com for Hopkins continuing medical education courses*. *Money paid to the institution.

\section{REFERENCES}

1. Milhorat TH, Chou MW, Trinidad EM, et al. Chiari I malformation redefined: clinical and radiographic findings for 364 symptomatic patients. Neurosurgery 1999;44:1005-17 CrossRef Medline

2. Speer MC, Enterline DS, Mehltretter L, et al. Review article: Chiari type I malformation with or without syringomyelia-prevalence and genetics. J Gen Couns 2003;12:297-311 CrossRef Medline

3. Strahle J, Muraszko KM, Kapurch J, et al. Chiari malformation type $\mathrm{I}$ and syrinx in children undergoing magnetic resonance imaging. J Neurosurg Pediatr 2011;8:205-13 CrossRef Medline

4. Ellenbogen RG, Armonda RA, Shaw DW, et al. Toward a rational treatment of Chiari I malformation and syringomyelia. Neurosurg Focus 2000;8:1-10 Medline

5. Moriwaka F, Tashiro K, Tachibana S, et al. Epidemiology of syringo- 
myelia in Japan: the nationwide survey [in Japanese]. Rinsho Shinkeigaku 1995;35:1395-97 Medline

6. Haines SJ, Berger M. Current treatment of Chiari I malformations types I and II: a survey of the Pediatric Section of the American Association of Neurological Surgeons. Neurosurgery 1991;28:353-57 CrossRef Medline

7. Yan $\mathrm{H}$, Zhu Z, Liu Z, et al. Diffusion tensor imaging in cervical syringomyelia secondary to Chiari I malformation: preliminary results. Spine (Phila Pa 1976) 2015;40:E381-87 CrossRef Medline

8. Koenigsberg RA, Vakil N, Hong TA, et al. Evaluation of platybasia with MR imaging. AJNR Am J Neuroradiol 2005;26:89-92 Medline

9. Besachio DA, Khaleel Z, Shah LM. Odontoid process inclination in normal adults and in an adult population with Chiari malformation type I. J Neurosurg Spine 2015;23:701-06 CrossRef Medline

10. Pang D, Thompson DN. Embryology and bony malformations of the craniovertebral junction. Childs Nerv Syst 2011;27:523-64 CrossRef Medline

11. Aitken LA, Lindan CE, Sidney S, et al. Chiari type I malformation in a pediatric population. Pediatr Neurol 2009;40:449-54 CrossRef Medline

12. Vega A, Quintana F, Berciano J. Basichondrocranium anomalies in adult Chiari type I malformation: a morphometric study. J Neurol Sci 1990;99:137-45 CrossRef Medline

13. Bollo RJ, Riva-Cambrin J, Brockmeyer MM, et al. Complex Chiari malformations in children: an analysis of preoperative risk factors for occipitocervical fusion. J Neurosurg Pediatr 2012;10:134-41 CrossRef Medline

14. Brockmeyer DL. The complex Chiari I: issues and management strategies. Neurol Sci 2011;32(suppl 3):S345-47 CrossRef Medline

15. Moore HE, Moore KR. Magnetic resonance imaging features of complex Chiari malformation variant of Chiari 1 malformation. Pediatr Radiol 2014;44:1403-11 CrossRef Medline

16. Sgouros S, Kountouri M, Natarajan K. Skull base growth in children with Chiari malformation type I. J Neurosurg 2007;107(3 suppl): 188-92 Medline

17. Bunck AC, Kroeger JR, Juettner A, et al. Magnetic resonance 4D flow analysis of cerebrospinal fluid dynamics in Chiari I malformation with and without syringomyelia. Eur Radiol 2012;22:1860-70 CrossRef Medline

18. Schuster JM, Zhang F, Norvell DC, et al. Persistent/recurrent syringomyelia after Chiari decompression: natural history and management strategies—a systematic review. Evid Based Spine Care J 2013; 4:116-25 CrossRef Medline

19. Clarke EC, Fletcher DF, Stoodley MA, et al. Computational fluid dynamics modelling of cerebrospinal fluid pressure in Chiari malformation and syringomyelia. J Biomech 2013;46:1801-09 CrossRef Medline 